\title{
Formose reaction controlled by boronic acid compounds
}

\author{
Toru Imai, Tomohiro Michitaka and Akihito Hashidzume*
}

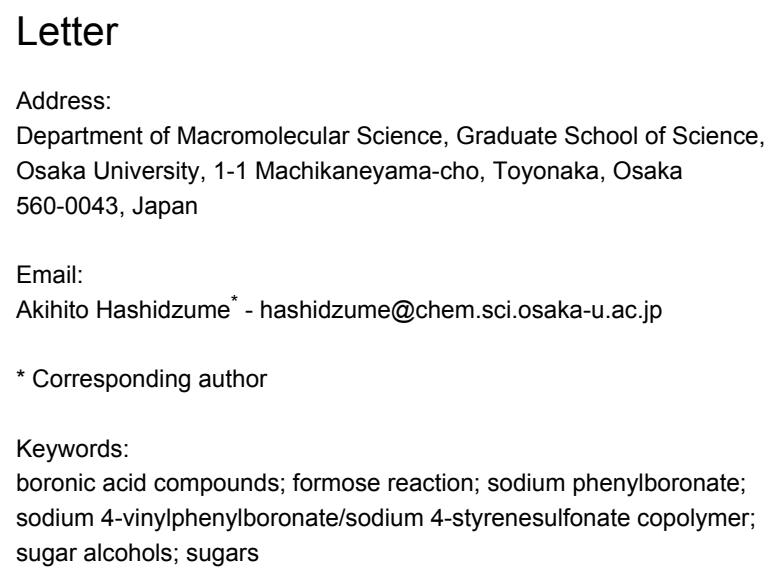

Beilstein J. Org. Chem. 2016, 12, 2668-2672.

doi:10.3762/bjoc. 12.263

Received: 26 August 2016

Accepted: 21 November 2016

Published: 08 December 2016

Associate Editor: H. Ritter

(c) 2016 Imai et al.; licensee Beilstein-Institut. License and terms: see end of document.

\begin{abstract}
Formose reactions were carried out in the presence of low molecular weight and macromolecular boronic acid compounds, i.e., sodium phenylboronate (SPB) and a copolymer of sodium 4-vinylphenylboronate with sodium 4-styrenesulfonate (pVPB/NaSS), respectively. The boronic acid compounds provided different selectivities; sugars of a small carbon number were formed favorably in the presence of SPB, whereas sugar alcohols of a larger carbon number were formed preferably in the presence of $\mathrm{pVPB} / \mathrm{NaSS}$.
\end{abstract}

\section{Findings}

When an aqueous solution of formaldehyde is warmed in the presence of a basic catalyst, a mixture of sugars and sugar alcohols, i.e., 'formose', is obtained. This reaction is called 'formose reaction' and was first reported by Butlerow, a Russian chemist, in 1861 [1]. Studies on formose reactions have revealed that the formose reaction consists of several elemental reaction steps, e.g., acyloin condensation, aldol reaction, retroaldol reaction, aldose-ketose isomerization, and Cannizzaro reaction, and the product, formose, is a complicated mixture of more than thirty species of sugars and sugar alcohols including non-natural ones, i.e., branched and L-isomers [2]. The formose reaction has being considered as a possible pathway for sugar formation under prebiotic conditions [3-5]. Some pioneering works from late 1970's to 1980 's have demonstrated that some sugars or sugar alcohols are selectively formed by optimizing the conditions of the formose reaction [6-15]. However, the selective formose reaction is still an important subject of investigation [16].

It is known that boronic acid compounds form esters with diols, e.g., sugars $[17,18]$. Boronic acid compounds may thus stabilize the sugars formed in the formose reaction [19]. Since the stability of boronic acid esters is dependent on the relative configuration of two hydroxy groups, i.e., the type of sugar [17,18], boronic acid compounds may provide some selectivity to formose reaction. In this study, we have thus carried out formose reactions in the presence of low molecular weight and macromolecular boronic acid compounds. 
Sodium phenylboronate (SPB) and a copolymer of sodium 4-vinylphenylboronate with sodium 4-styrenesulfonate (pVPB/ $\mathrm{NaSS}$ ) were used as a low molecular weight boronic acid compound and a boronic acid polymer, respectively (Scheme 1). The copolymer was prepared by radical copolymerization at a molar ratio of 1:10 in monomer feed. The formose reaction was carried out using a solution containing $200 \mathrm{mM}$ formaldehyde and $20 \mathrm{mM}$ calcium hydroxide at $60^{\circ} \mathrm{C}$. Fructose or glyceraldehyde was employed as a cocatalyst because the formose reaction did not proceed in the presence of a boronic acid compound without cocatalyst. The conversions of formose reactions were determined by the acetylacetone method [20,21]. Figure 1a shows a typical example of the time-conversion plots for formose reactions in the presence of SPB. In the presence of $5 \mathrm{mM}$ SPB, the time-conversion plots are almost the same as those for formose reactions in the absence of boronic acid compounds, indicative of no effect of SPB at a lower concentration. At 8,10 , and $12 \mathrm{mM}$, the conversion shows a small onset and then increases gradually. After ca. $30 \mathrm{~min}$, the conversion starts to increase significantly and then reaches a quantitative conversion. At concentrations $\geq 15 \mathrm{mM}$, the conversion is ca. $30 \%$ even after $90 \mathrm{~min}$. These data indicate that SPB retards the formose reaction. Figure $1 \mathrm{~b}$ shows the time-conversion plots for formose reactions carried out in the presence of $\mathrm{pVPB} /$ NaSS. The formose reaction is retarded more significantly at higher concentrations. At $30 \mathrm{~g} \mathrm{~L}^{-1}$, the conversion was ca. $40 \%$ even after $180 \mathrm{~min}$, indicating that $\mathrm{pVPB} / \mathrm{NaSS}$ also retards the formose reaction. Figure S1 in Supporting Information File 1<smiles>O[B-](O)(O)c1ccccc1</smiles>

SPB

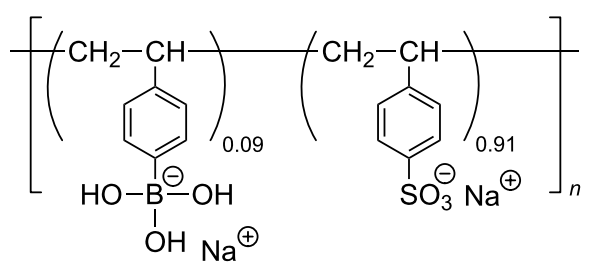

$$
\begin{gathered}
M_{\mathrm{w}}=1.4 \times 10^{4}, M_{\mathrm{w}} / M_{\mathrm{n}}=3.31 \\
\text { pVPB } / \text { NaSS }
\end{gathered}
$$

Scheme 1: Structures of the boronic acid compounds used in this study (i.e., SPB and pVPB/NaSS). compares the time-conversion data for SPB and $\mathrm{pVPB} / \mathrm{NaSS}$. This figure indicates that $\mathrm{pVPB} / \mathrm{NaSS}$ retards the formose reaction more significantly than SPB does at similar concentrations of boronic acid residues. In a separate experiment, the formose reaction did not proceed significantly in the presence of $100 \mathrm{~g} \mathrm{~L}^{-1}$ poly(sodium 4-styrenesulfonate) presumably because sulfonate residues capture calcium ions. These observations indicate that the stronger retardation effect of $\mathrm{pVPB} / \mathrm{NaSS}$ is ascribable to both boronic acid and sulfonate residues.

(a)

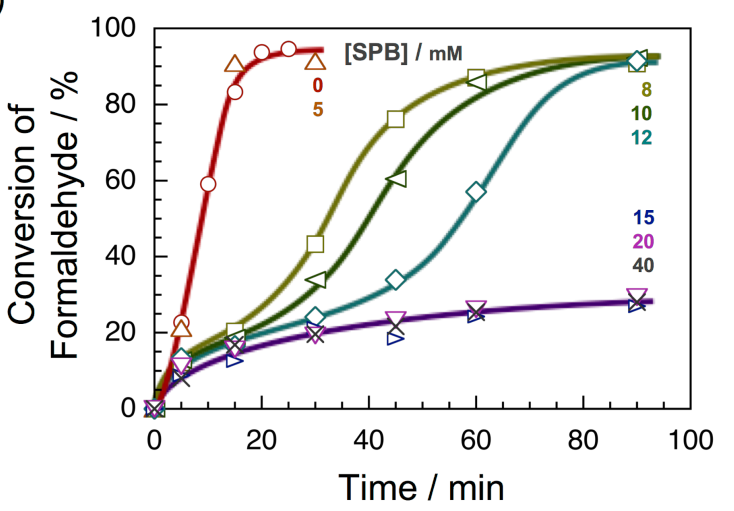

(b)

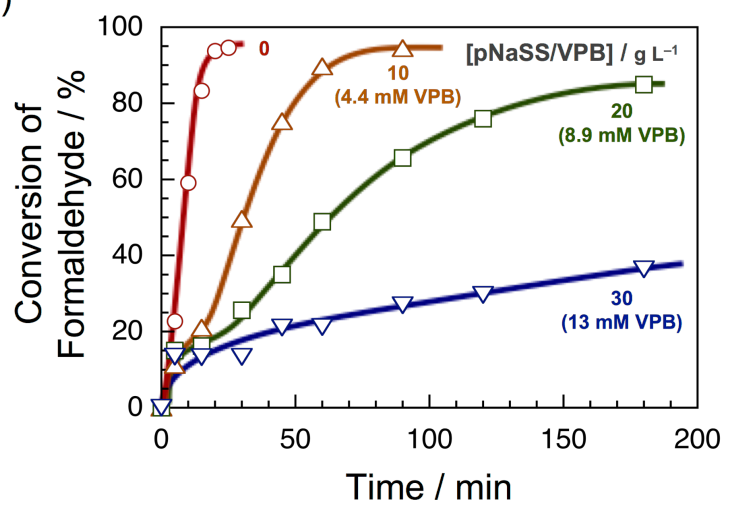

Figure 1: Time-conversion plots for the formose reaction using $200 \mathrm{mM}$ formaldehyde, $20 \mathrm{mM}$ calcium hydroxide, and $6.9 \mathrm{mM}$ cocatalyst in the presence of SPB (a) and pVPB/NaSS (b); fructose and glyceraldehyde was used as a cocatalyst for SPB and pVPB/NaSS, respectively. The curves are drawn as a guide for the eye.

The product was purified by dialysis against water and treatment with ion-exchange resins, and then recovered by freezedrying, and characterized by high-performance liquid chromatography (HPLC) using an amino column and a mixed solvent of water and acetonitrile, as can be seen in Figure 2. As reference, this figure also contains an HPLC chart for standard samples, i.e., two-, three-, four-, five-, and six-carbon sugar alcohols (ethylene glycol, glycerol, erythritol, D-arabinitol, and D-mannitol, respectively) (Figure 2a). In Figure $2 b-d$, the 
signals at ca. 5 min may be due to impurities, e.g., inorganic salts. As can be seen in Figure 2b, the HPLC chart for the formose reaction in the absence of boronic acid compounds contains a number of signals in a wide range of elution time, indicative for the formation of a complicated mixture. On the other hand, the HPLC chart for SPB indicates a broad signal in the region of smaller carbon numbers (Figure 2c), and the chart for $\mathrm{pVPB} / \mathrm{NaSS}$ exhibits signals in the region of larger carbon numbers (Figure 2d). These data are suggestive of modest selectivity of the formose reaction in the presence of boronic acid compounds.

Figure 3 compares ${ }^{1} \mathrm{H}$ and ${ }^{13} \mathrm{C}$ NMR spectra for the products obtained in the presence of SPB and pVPB/NaSS. The ${ }^{1} \mathrm{H}$ and ${ }^{13} \mathrm{C}$ NMR spectra for SPB exhibit broad signals, similar to those for a formose reaction without boronic acid compounds. Since it was difficult to remove SPB from the reaction mixture, the NMR sample contained SPB, which might cause the broadening of NMR signals presumably because of equillibria of the formation of boronic acid esters. On the other hand, the ${ }^{1} \mathrm{H}$ and ${ }^{13} \mathrm{C}$ NMR spectra for $\mathrm{pVPB} / \mathrm{NaSS}$ show well-resolved signals, indicating that the formose reaction provides products with a significant selectivity in the presence of $\mathrm{pVPB} / \mathrm{NaSS}$. Figure 4 displays typical examples of electrospray ionization mass spectrometry (ESIMS) data for the products of the formose reaction in the presence of SPB and $\mathrm{pVPB} / \mathrm{NaSS}$. The spectrum for SPB shows a series of signals at $m / z=215,245$, and 275, indicating an interval of 30 mass units, which corresponds to the formalde-

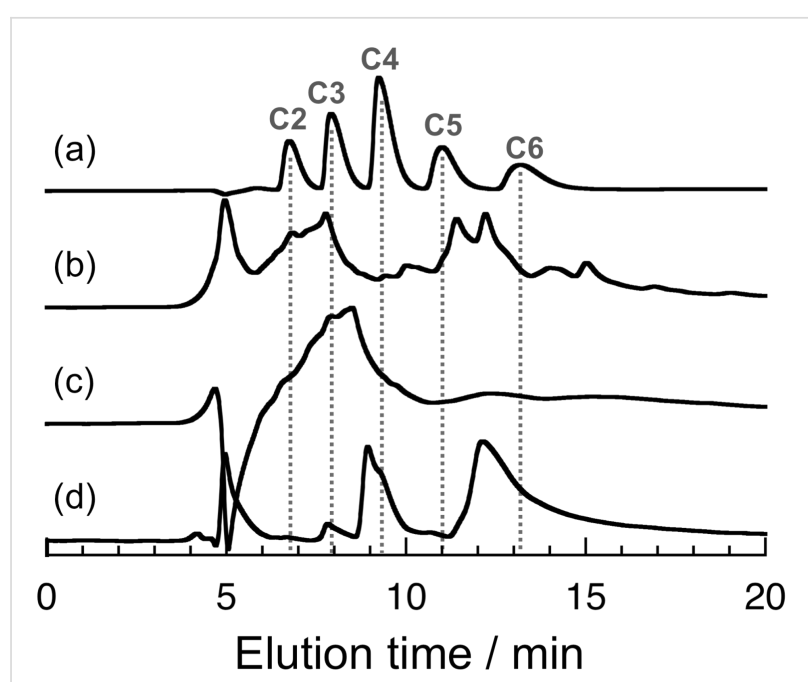

Figure 2: HPLC charts for standard samples (a) and products of the formose reaction in the absence (b) and presence of $10 \mathrm{mM} \mathrm{SPB} \mathrm{(c)}$ and $20 \mathrm{~g} \mathrm{~L}^{-1} \mathrm{pVPB} / \mathrm{NaSS}$ (d); the reaction times were 20,90 , and $180 \mathrm{~min}$ for (b), (c), and (d), respectively.

hyde unit. These signals are ascribable to potassium adducts of boronic acid esters formed from SPB and three-, four-, and fivecarbon sugars. On the other hand, the spectrum for $\mathrm{pVPB} / \mathrm{NaSS}$ contains a series of signals at $m / z=145,175,205,235$, and 265. These signals are assignable to sodium adducts of four to eight carbon sugar alcohols. On the basis of these characterization data, we can conclude that the formose reaction in the presence of SPB produces favorably sugars of a small carbon num-
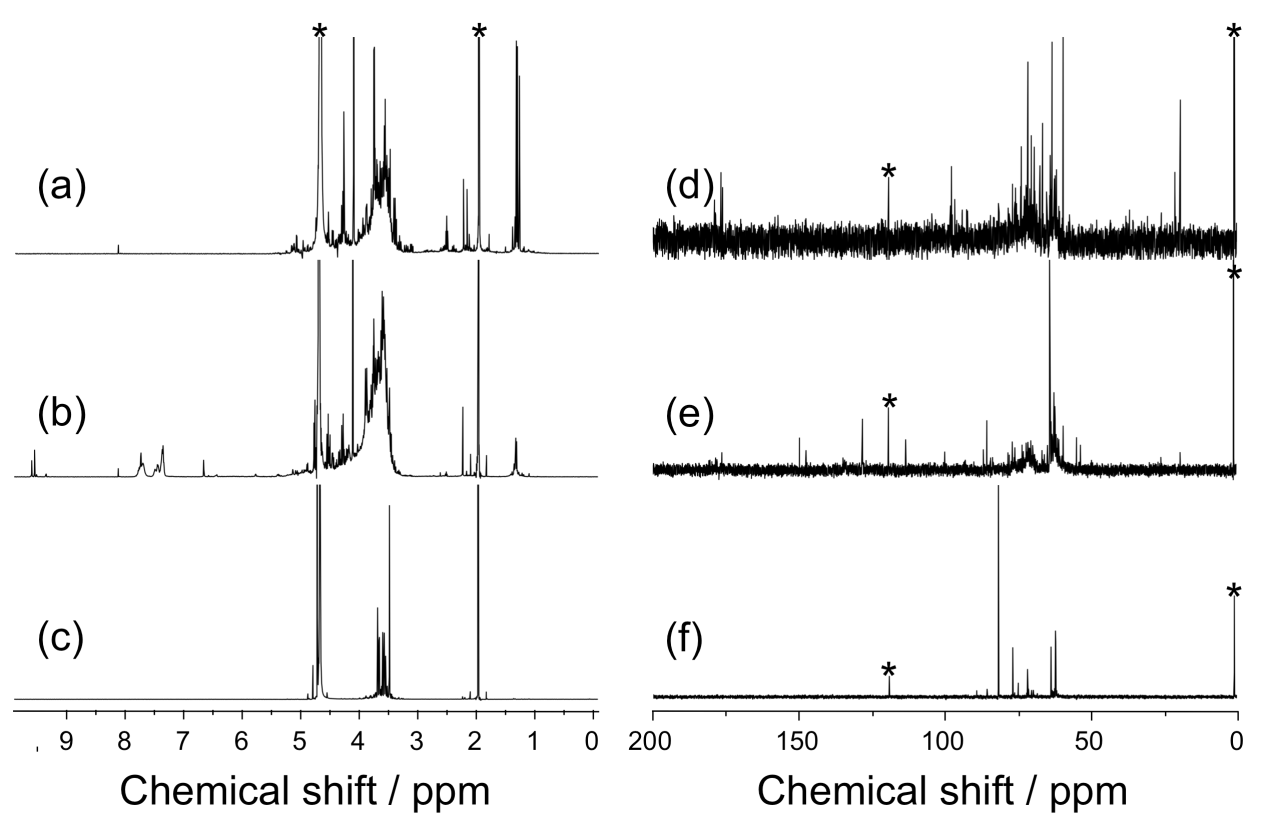

Figure 3: ${ }^{1} \mathrm{H}(\mathrm{a}, \mathrm{b}$, and $\mathrm{c})$ and ${ }^{13} \mathrm{C}$ NMR spectra $(\mathrm{d}, \mathrm{e}$, and $\mathrm{f})$ for the products of the formose reaction in the absence (a and $\left.\mathrm{d}\right)$ and presence of $10 \mathrm{mM}$ SPB (b and e) and $20 \mathrm{~g} \mathrm{~L}^{-1} \mathrm{pVPB} / \mathrm{NaSS}$ (c and f); the reaction times were 20, 90, and $180 \mathrm{~min}$ for (a and d), (b and e), and (c and f), respectively. Asterisks denote the signals of the internal standard, i.e., acetonitrile. 
ber whereas the formose reaction in the presence of $\mathrm{pVPB} /$ NaSS produces preferably sugar alcohols of a larger carbon number.

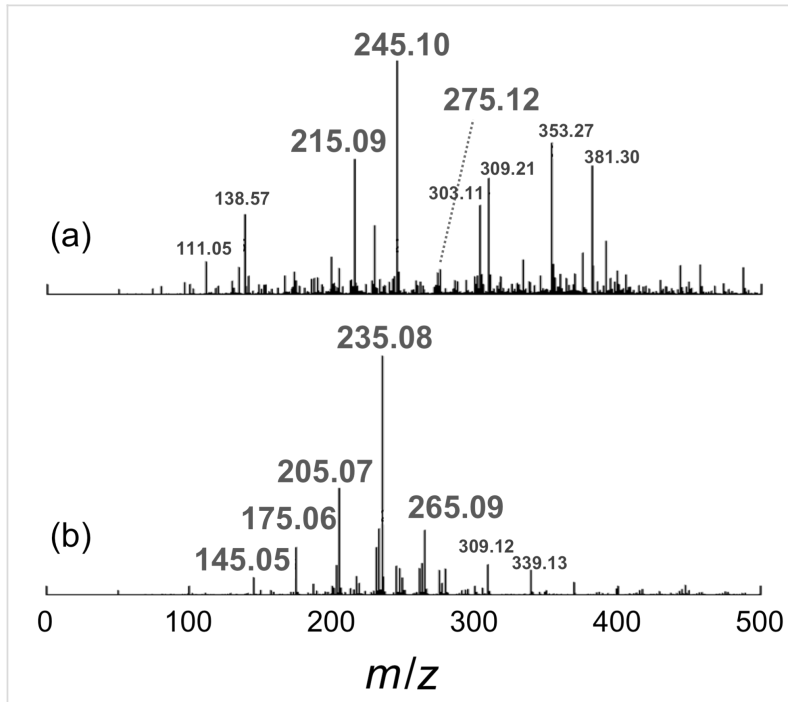

Figure 4: ESIMS data for the products of the formose reaction in the presence of $10 \mathrm{mM} \mathrm{SPB} \mathrm{(a)} \mathrm{and} 20 \mathrm{~g} \mathrm{~L}^{-1} \mathrm{pVPB} / \mathrm{NaSS}$ (b); the reaction times were 90 and 180 min for (a) and (b), respectively.

Here we discuss the effect of SPB and $\mathrm{pVPB} / \mathrm{NaSS}$ on formose reaction. As shown in Figure 1, both SPB and pVPB/NaSS retarded the formose reaction, indicating that boronic acid compounds form esters with the sugars and sugar alcohols formed and the esters somehow protect the sugars or sugar alcohols from further reaction. The ESIMS data of products elucidated that the formose reactions in the presence of SPB and pVPB/ NaSS exhibited different selectivities; sugars of a small carbon number were formed preferably in the presence of SPB, whereas sugar alcohols of a larger carbon number were obtained favorably in the presence of $\mathrm{pVPB} / \mathrm{NaSS}$ (Figure 4). The selectivity of products should be dependent on the stability of esters. It is thus likely that SPB forms rather stable esters with sugars of a small carbon number and $\mathrm{pVPB} / \mathrm{NaSS}$ forms relatively stable esters with sugar alcohols of a larger carbon number. The detailed mechanism of selective formose reaction assisted with boronic acid compounds should be investigated in the near future.

In order to control the formose reaction, we carried out formose reactions in the presence of $\mathrm{SPB}$ and $\mathrm{pVPB} / \mathrm{NaSS}$. The time-conversion data indicate that SPB and $\mathrm{pVPB} / \mathrm{NaSS}$ retarded the formose reaction. The characterization data by HPLC, NMR, and ESIMS for the products indicated that sugars of a small carbon number were formed favorably in the presence of SPB, whereas sugar alcohols of a larger carbon number were formed preferably in the presence of $\mathrm{pVPB} / \mathrm{NaSS}$.

\section{Supporting Information}

\section{Supporting Information File 1}

Experimental section and time-conversion plots for formose reactions in the presence of SPB and $\mathrm{pVPB} / \mathrm{NaSS}$. [http://www.beilstein-journals.org/bjoc/content/ supplementary/1860-5397-12-263-S1.pdf]

\section{Acknowledgements}

The authors would like to express their acknowledgment to Professor Takahiro Sato, Department of Macromolecular Science, Graduate School of Science, Osaka University, for his technical support and fruitful discussion. This work was partly supported by JSPS Kakenhi Grant Numbers JP23550137, JP26288061.

\section{References}

1. Butlerow, A. Justus Liebigs Ann. Chem. 1861, 120, 295-298. doi:10.1002/jlac.18611200308

2. Mizuno, T.; Weiss, A. H. Adv. Carbohydr. Chem. Biochem. 1974, 29, 173-227. doi:10.1016/S0065-2318(08)60250-4

3. Gardner, P. M.; Winzer, K.; Davis, B. G. Nat. Chem. 2009, 1, 377-383. doi:10.1038/nchem.296

4. Harman, C. E.; Kasting, J. F.; Wolf, E. T. Origins Life Evol. Biospheres 2013, 43, 77-98. doi:10.1007/s11084-013-9332-7

5. Kebukawa, Y.; Kilcoyne, A. L. D.; Cody, G. D. Astrophys. J. 2013, 771, No. 19. doi:10.1088/0004-637X/771/1/19

6. Shigemasa, Y.; Nagae, O.; Sakazawa, C.; Nakashima, R.; Matsuura, T. J. Am. Chem. Soc. 1978, 100, 1309-1310. doi:10.1021/ja00472a056

7. Shigemasa, Y.; Kawahara, M.; Sakazawa, C.; Nakashima, R.; Matsuura, T. J. Catal. 1980, 62, 107-116. doi:10.1016/0021-9517(80)90426-1

8. Shigemasa, Y.; Akagi, S.; Nakashima, R.; Saito, S. Carbohydr. Res. 1980, 80, C1-C3. doi:10.1016/S0008-6215(00)85330-6

9. Shigemasa, Y.; Hamada, T.; Hirabayashi, M.; Waki, E.; Nakashima, R.; Harada, K.; Takeda, N.; Suzuki, M. Chem. Lett. 1981, 10, 899-902. doi:10.1246/cl.1981.899

10. Shigemasa, Y.; Oogaki, K.; Ueda, N.; Hakashima, R.; Harada, K.; Takeda, N.; Suzuki, M.; Saito, S. J. Carbohydr. Chem. 1983, 1, 325-330. doi:10.1080/07328308208085105

11. Shigemasa, Y.; Sasaki, Y.; Ueda, N.; Nakashima, R. Bull. Chem. Soc. Jpn. 1984, 57, 2761-2767. doi:10.1246/bcsj.57.2761 12. Shigemasa, Y.; Ueda, T.; Saimoto, H. Bull. Chem. Soc. Jpn. 1990, 63, 389-394. doi:10.1246/bcsj.63.389

13. Matsumoto, T.; Komiyama, M.; Inoue, S. Chem. Lett. 1980, 9 , 839-842. doi:10.1246/cl.1980.839

14. Matsumoto, T.; Inoue, S. J. Chem. Soc., Perkin Trans. 11982 , 1975-1979. doi:10.1039/p19820001975

15. Matsumoto, T.; Yamamoto, H.; Inoue, S. J. Am. Chem. Soc. 1984, 106, 4829-4832. doi:10.1021/ja00329a031

16. Delidovich, I. V.; Simonov, A. N.; Taran, O. P.; Parmon, V. N. ChemSusChem 2014, 7, 1833-1846. doi:10.1002/cssc.201400040 
17. James, T. D. In Boronic Acids. Preparation, Applications in Organic Synthesis and Medicine; Hall, D. G., Ed.; Wiley-VCH: Weinheim, Germany, 2005; pp 441-479.

18. James, T. D.; Phillips, M. D.; Shinkai, S. Boronic Acids in Saccharide Recognition; The Royal Society of Chemistry: Cambridge, UK, 2006.

19. Ricardo, A.; Carrigan, M. A.; Olcott, A. N.; Benner, S. A. Science 2004, 303, 196. doi:10.1126/science. 1092464

20. http://www.maff.go.jp/nval/kijyun/pdf/ST06130.PDF.

21. Maruo, Y. Y.; Nakamura, J.; Uchiyama, M. Talanta 2008, 74, 1141-1147. doi:10.1016/j.talanta.2007.08.017

\section{License and Terms}

This is an Open Access article under the terms of the Creative Commons Attribution License

(http://creativecommons.org/licenses/by/4.0), which permits unrestricted use, distribution, and reproduction in any medium, provided the original work is properly cited.

The license is subject to the Beilstein Journal of Organic Chemistry terms and conditions:

(http://www.beilstein-journals.org/bjoc)

The definitive version of this article is the electronic one which can be found at: doi:10.3762/bjoc. 12.263 